\title{
Impact of Transcranial Direct Current Stimulation on Cognitive Function, Brain Functional Segregation, and Integration in Patients with Mild Cognitive Impairment According to Amyloid-Beta Deposition and $A P O E$ \&4-Allele: A Pilot Study
}

\author{
Dong-Woo Kang ${ }^{1}{ }^{\mathbb{D}}$, Sheng-Min Wang ${ }^{2}$, Tae-Yeong $\mathrm{Kim}^{3}{ }^{3}$, Donghyeon $\mathrm{Kim}^{3}{ }^{3}$, Hae-Ran Na ${ }^{2}$, Nak-Young Kim ${ }^{4}$, \\ Chang-Uk Lee ${ }^{1}$ and Hyun-Kook Lim ${ }^{2, *(D)}$ \\ 1 Department of Psychiatry, Seoul St. Mary's Hospital, College of Medicine, The Catholic University of Korea, \\ Seoul 06591, Korea; kato7@hanmail.net (D.-W.K.); jihan@catholic.ac.kr (C.-U.L.) \\ 2 Department of Psychiatry, Yeouido St. Mary's Hospital, College of Medicine, The Catholic University of \\ Korea, Seoul 07345, Korea; smwang11@naver.com (S.-M.W.); haeranna@gmail.com (H.-R.N.) \\ 3 Research Institute, NEUROPHET Inc., Seoul 06247, Korea; ty.kim@neurophet.com (T.-Y.K.); \\ donghyeon.kim@neurophet.com (D.K.) \\ 4 Department of Psychiatry, Keyo Hospital, Uiwang 16062, Korea; nakyoung17@gmail.com \\ * Correspondence: drblues@catholic.ac.kr
}

\section{check for}

updates

Citation: Kang, D.-W.; Wang, S.-M.; Kim, T.-Y.; Kim, D.; Na, H.-R.; Kim,

N.-Y.; Lee, C.-U.; Lim, H.-K. Impact of

Transcranial Direct Current

Stimulation on Cognitive Function,

Brain Functional Segregation, and Integration in Patients with Mild Cognitive Impairment According to Amyloid-Beta Deposition and APOE \&4-Allele: A Pilot Study. Brain Sci. 2021, 11, 772. https://doi.org/ 10.3390/brainsci11060772

Received: 15 May 2021

Accepted: 7 June 2021

Published: 10 June 2021

Publisher's Note: MDPI stays neutral with regard to jurisdictional claims in published maps and institutional affiliations.

Copyright: (c) 2021 by the authors. Licensee MDPI, Basel, Switzerland. This article is an open access article distributed under the terms and conditions of the Creative Commons Attribution (CC BY) license (https:/ / creativecommons.org/licenses/by/ $4.0 /)$.

\begin{abstract}
Anodal transcranial direct current stimulation (anodal-tDCS) is known to improve cognition and normalize abnormal network configuration during resting-state functional magnetic resonance imaging (fMRI) in patients with mild cognitive impairment (MCI). We aimed to evaluate the impact of sequential anodal-tDCS on cognitive functions, functional segregation, and integration parameters in patients with $\mathrm{MCI}$, according to high-risk factors for Alzheimer's disease (AD): amyloid-beta $(\mathrm{A} \beta)$ deposition and $A P O E$ \&4-allele status. In 32 patients with $\mathrm{MCI}\left({ }^{18} \mathrm{~F}\right]$ flutemetamol-: $n=10$, [ $\left.{ }^{18} \mathrm{~F}\right]$ flutemetamol+: $n=22 ;$ APOE $\left.\varepsilon 4-: n=13, A P O E \varepsilon 4+: n=19\right)$, we delivered anodal-tDCS ( $2 \mathrm{~mA} /$ day, five times/week, for 2 weeks) over the left dorsolateral prefrontal cortex and assessed the neuropsychological test battery and resting-state fMRI measurements before and after 2 weeks stimulation. We observed a non-significant impact of an anodal-tDCS on changes in neuropsychological battery scores between $\mathrm{MCI}$ patients with and without high-risk factors of $\mathrm{AD}, \mathrm{A} \beta$ retention and $A P O E \varepsilon 4$-allele. However, there was a significant difference in brain functional segregation and integration parameters between MCI patients with and without AD high-risk factors. We also found a significant effect of tDCS-by-APOE $\varepsilon 4$-allele interaction on changes in the functional segregation parameter of the temporal pole. In addition, baseline $A \beta$ deposition significantly associated negatively with change in global functional integrity of hippocampal formation. Anodal-tDCS might help to enhance restorative and compensatory intrinsic functional changes in MCI patients, modulated by the presence of $\mathrm{A} \beta$ retention and the $A P O E \varepsilon 4$-allele.
\end{abstract}

Keywords: amyloid beta deposition; $A P O E$ \&4-allele; mild cognitive impairment; transcranial direct current stimulation

\section{Introduction}

Alzheimer's disease (AD) is a leading cause of dementia and imposes a marked social and economic burden. Mild cognitive impairment (MCI), a prodromal AD stage, involves subjective and objective decline in cognitive function, but preservation of the independent daily living ability [1]. Since $10-15 \%$ of MCI patients convert to dementia annually, various attempts have been made to delay or prevent the transition to dementia at this stage [2]. Although therapeutic attempts, such as cognitive intervention [3], regular physical exercise [4], and dietary intervention have shown some positive results for changes in cognitive function and biomarkers [5], additional evidence is needed for these interventions to be 
established as an $\mathrm{AD}$ prevention strategy. Furthermore, it is often difficult for $\mathrm{MCI}$ patients to perform preventive interventions with increased complexity and to maintain consistency for a significant period [6]. Therefore, the importance of an intervention that can be applied in a simple and fixed manner and maintained consistently for a certain period of time is emphasized.

In this regard, noninvasive brain stimulation has been proposed as a potential treatment option in the course of AD [7]. Transcranial direct current stimulation (tDCS), a type of noninvasive brain stimulation, modulates the excitability of cortical neurons depending on the current flow direction [8]. Moreover, tDCS has synaptic after-effects through long-term potentiation and alter oscillatory brain activity and functional connectivity patterns [9].

In some previous studies, AD patients showed improvement in the MMSE score [10], recognition memory [11], and global cognitive performance after tDCS was applied [12], while other studies found no significant difference in cognitive function compared with the sham group [13]. In these studies, the dorsolateral prefrontal cortex (DLPFC) has been most frequently targeted, and tDCS was applied in single or multiple sessions. It has been reported that excitatory input in the DLPFC affects memory performances, boosting parietal capacity and playing a compensatory role against a decline in medial-temporal function $[14,15]$. There is a relative paucity of studies investigating the impact of tDCS on cognitive performance in patients with MCI. Prior research has shown an improvement in word retrieval performance after single-session anodal-tDCS application to the left ventral inferior frontal gyrus of patients with MCI [16]. However, another study found no significant difference in cognitive test battery scores after nine sessions of anodal-tDCS of the left DLPFC in patients with MCI [17].

Resting-state functional MRI (rs-fMRI) reveals intrinsic brain activity in the resting state and can approach functional segregation and integration by evaluating the fractional amplitude of low-frequency fluctuation (fALFF) and degree centrality (DC) [18]. Previous studies have shown that changes and disruptions in functional segregation and integration are associated with AD progression [19,20]. Additionally, the default mode network (DMN) is a characteristic network of increased intrinsic brain activity during the resting state [21], and aberrant changes in this network have been demonstrated to reflect deterioration of AD [22]. Direct current stimulation has been documented to modulate the DMN and affect changes in functional segregation and integration parameters [22,23]. However, few studies have evaluated the impact of tDCS on functional segregation and integration of intrinsic brain activity in the prodromal stage of AD.

Amyloid-beta $(A \beta)$ retention and $A P O E \varepsilon 4$ genotype, which are representative factors affecting the progression and prognosis of $\mathrm{AD}$, have been reported to affect the neuronal activity and cognitive decline significantly [24-27]. Furthermore, these AD risk factors have been demonstrated to affect the outcomes of preventive attempts in the prodromal stage of AD $[28,29]$. Nevertheless, few studies have examined the effects of tDCS on cognitive and functional brain changes according to these AD risk factors in the $\mathrm{MCI}$ stage and there is little evidence for a precision medicine approach to the tDCS in the prodromal stage of $\mathrm{AD}$.

Consequently, this study evaluated the impact of anodal-tDCS on cognitive performance and functional segregation and integration parameters in $\mathrm{MCI}$ patients, depending on $\mathrm{A} \beta$ deposition and $A P O E$ \&4-allele status. We hypothesized that there would be a significant difference in changes in cognitive function and intrinsic brain activity between MCI patients with and without AD risk factors after multiple sessions of anodal-tDCS. Furthermore, we also explored whether the interaction between anodal-tDCS application and $\mathrm{AD}$ risk factors affects changes in cognitive function and intrinsic brain activity in the prodromal stage of AD.

\section{Materials and Methods}

\subsection{Participants}

Thirty-two MCI patients were recruited from the Brain Health Center, Yeoui-do St. Mary's Hospital, College of Medicine, The Catholic University of Korea, Republic of 
Korea, from May 2020 to December 2020. The study was conducted in accordance with the Declaration of Helsinki and was approved by the Institutional Review Board of the Catholic University of Korea. Informed and written consent was obtained from all participants.

The cognitive functions of all subjects were assessed with the Korean version of the Consortium to Establish a Registry for Alzheimer's Disease (CERAD-K) [30], which included a verbal fluency (VF) test, the 15-item Boston Naming Test (BNT), the Korean version of the Mini-Mental State Examination (MMSE-K) [31], word list memory (WLM), word list recall (WLR), word list recognition (WLRc), constructional praxis (CP), and constructional recall (CR) assessments. Additionally, total scores of memory domains (TM) were obtained by summing the CERAD-K, WLM, WLR, WLRc, and CR scores. Total CERAD-K scores were calculated by summing all CERAD-K subcategory scores, excluding the MMSE-K score.

Patients with amnestic MCI met Peterson's criteria [32]. Inclusion criteria for MCI participants are described in a detail in the Supplementary Material. All subjects were evaluated at the Brain Health Center by an experienced psychiatrist and a psychologist. Details surrounding the usage of specific tests and the reviewing process are described in the Supplementary Material.

\subsection{Experimental Design}

In this study, patients received 10 tDCS sessions (five times/week for 2 weeks: 10 sessions). The participants were assessed with the CERAD-K neuropsychological battery and underwent resting-state fMRI within 2 weeks before the first tDCS session and after the 10 th session. Subjects underwent $\left[{ }^{18} \mathrm{~F}\right]$ flutemetamol (FMM) positron emission tomography-computed tomography (PET-CT) and APOE genotyping within 4 weeks before the first $\mathrm{tDCS}$ session. In addition, participants and the psychologists who performed the neuropsychological battery were blinded to the results of amyloid-PET and APOE genotyping.

\section{3. tDCS Application}

A constant direct current (2 mA, $20 \mathrm{~min}$ ) was administered by an MRI-compatible stimulator (YDS-301N, YBrain, Seoul, Republic of Korea). The anode was attached over the left DLPFC (F3 in the International 10/20 electroencephalogram system). The cathode was positioned over the right supraorbital region. The electrodes touched a water-soaked sponge (disc type, radius $=3 \mathrm{~cm}$ ) placed on the scalp. For the subject to apply the device accurately, staff skilled in the use of the device visited the patient's residence for each stimulus session to guide device application.

\section{4. fMRI Data Acquisition and Data Processing}

Imaging data were collected by the Department of Radiology of Yeouido Saint Mary's Hospital at the Catholic University of Korea using a 3-T Siemens Skyra MRI machine and a 32-channel Siemens head coil (Siemens Medical Solutions, Erlangen, Germany). Parameters of structural and functional MRI data acquisition are described in the Supplementary Material.

We used the Data Processing Assistant for Resting-State fMRI (DPARSF, GNU GENERAL PUBLIC LICENSE, Beijing, China) [33], which is based on Statistical Parametric Mapping (SPM, http:/ /www.fil.ion.ucl.ac.uk/spm, Wellcome Centre for Human Neuroimaging, London, UK), to preprocess the fMR images. Slice timing and realignment for motion corrections were performed on the images. Subjects with excessive head motion (cumulative translation or rotation $>2 \mathrm{~mm}$ or $2^{\circ}$ ) were excluded. To prevent group-related differences caused by microscopic head motion, framewise displacement (FD) was compared between the groups. Mean FD scores did not differ between groups $(p>0.05$, 2-sample $t$ test). For spatial registration, T1-weighted images were co-registered to the mean rsfMRI image based on rigid-body transformation. For spatial normalization, the International Consortium for Brain Mapping template was applied (resampling voxel size 
$=3 \times 3 \times 3 \mathrm{~mm}$ ) and fitted to the "East Asian brain". After this, the functional images were spatially smoothed with a $6 \mathrm{~mm}$ full width at half maximum Gaussian kernel.

We further processed our functional data to fit them to fALFF and DC analysis with DPARSF. Linear trends were removed from the functional images, and data were filtered with a temporal band-pass of $0.01-0.08 \mathrm{~Hz}$, to reduce low-frequency drift as well as physiological high-frequency respiratory and cardiac noise. Several nuisance covariates were regressed out, including six head motion parameters and signals from the WM and CSF.

\section{5. fALFF and DC Analysis}

To measure regional intrinsic brain activities in the resting state, fALFF was computed using individual preprocessed data [19]. The process of calculating fALFF is described in detail in the Supplementary Material. This fALFF calculation was repeated for each voxel in the whole brain to create a fALFF map for each participant, which was used in statistical analysis.

The DC was computed as the number of significant correlations (binarized) or as the sum of the weights of the significant connections (weighted) for each voxel (a threshold of $r>0.25, p<0.05$ ). The map of the connectivity was then standardized by conversion to $z$ scores, so that maps across participants could be averaged and compared. DC represents the most local and directly quantifiable centrality measure and has been widely used to examine node characteristics of intrinsic network connectivity [34]. Within the DMN, the DC value of a node indicates its connectivity strength to all the other nodes and reflects its importance in functional integration. Additionally, the fALFF and DC were calculated in 11 predefined regions-of-interest (ROIs) in the DMN and were used in statistical analysis (Table S1 in the Supplementary Materials) [35]. Moreover, whole-brain voxel-wise analysis of fALFF and DC was also performed.

\section{6. $\left[{ }^{18}\right.$ F] Flutemetamol PET-CT Image Acquisition, Assessments, and SUVR Calculations}

$\left[{ }^{18} \mathrm{~F}\right]$ FMM was manufactured, and FMM-PET data were collected and analyzed as described previously [36]. MRI of each participant was used to co-register and define the ROIs, and correct partial volume effects arising from the expansion of cerebrospinal spaces accompanying cerebral atrophy. We used a standardized uptake value ratio (SUVR) at 90-min post-injection to analyze the FMM PET data, using the pons ROI as the reference. Global A $\beta$ burden was expressed as the average SUVR of the mean for the six cortical ROIs, including the frontal, superior parietal, lateral temporal, striatum, anterior, and posterior cingulate cortex/precuneus ROIs. We used a cut-off for "high" or 'low' neocortical SUVR of 0.62 , consistent with cut-off values used in previous FMM PET study [36].

\subsection{Statistical Analysis}

Statistical analyses for demographic data were performed using R software (version 2.15.3). Assumptions of normality were tested for continuous variables using the Kolmogorov-Smirnov test; all data demonstrated a normal distribution. Two sample t-tests and chi-square $\left(\chi^{2}\right)$ tests were used to probe for differences in demographic variables, clinical data, cognitive function, and fMRI measurements between MCI patients with and without $\mathrm{A} \beta$ deposition and the APOE $\varepsilon 4$-allele. Cognitive function and fMRI parameters (fALFF and DC in ROIs of the DMN) over 10 sessions were analyzed for change with a repeated-measures analysis of variance (ANOVA) with time (pre-tDCS and post-tDCS) as repeated-measures factor and the presence of $\mathrm{A} \beta$ deposits and the APOE $\varepsilon 4$-allele as the between-subject factor, with adjustments for age, sex, and years of education. Multiple regression analysis was performed to evaluate the association between baseline $\left[{ }^{18} \mathrm{~F}\right] \mathrm{FMM}$ SUVR $_{\mathrm{PONS}}$ and change in cognitive function and rs-fMRI measurements (fALFF and DC in ROIs of DMN), adjusting for age, sex, education years, and APOE $\varepsilon 4$-allele. Each variable was z-transformed using the mean and standard deviation. All statistical analyses used a two-tailed $p$-value $<0.05$ to define statistical significance. 
Additionally, to observe the effects of tDCS-by-group interaction on AALFF and DC, a mixed analysis on a voxel-by-voxel basis, with groups ( $A P O E \varepsilon 4$-allele carrier vs. noncarrier; positive vs. negative for $A \beta$ retention) as between-subject factors and tDCS (pretDCS vs. post-tDCS) as within-subject factors was performed on a brain mask. Age, sex, and years of education were included as covariates in the statistical tests. We designed a mixed analysis based on the SPM 12. An F-contrast was designed for the interaction effect analysis. Furthermore, paired t-tests were performed between pre-tDCS and post-tDCS on the individual $\mathrm{z}$ maps of fALFF and DC in each sub-group, respectively (negative or positive for $\mathrm{A} \beta$ retention; $A P O E \varepsilon 4$-allele carrier or non-carrier). All statistical maps were corrected for multiple comparisons by Gaussian random field (GRF) correction combining the voxel $p$-value $<0.001$ and cluster level $<0.05$ in DPABI_V5.1_201201 (http://rfmri.org/dpabi, GNU GENERAL PUBLIC LICENSE, Beijing, China) [37].

\section{Results}

\subsection{Baseline Demographic and Clinical Data}

Table 1 shows the baseline demographic and clinical data for MCI patients classified by the presence of $\mathrm{A} \beta$ deposits and the $A P O E \varepsilon 4$-allele. MCI patients with $\mathrm{A} \beta$ deposits showed higher years of education than those without $A \beta$ accumulation (Table 1A). The ratio of $A P O E \varepsilon 4$ carriers was significantly higher in the group with $\mathrm{A} \beta$ deposits. This group displayed higher average $\mathrm{SUVR}_{\mathrm{PONS}}$ than that without $\mathrm{A} \beta$ deposits (Table $1 \mathrm{~A}$ ).

Table 1. Demographic and clinical characteristics of study participants categorized by (A) A $\beta$ deposits and (B) APOE \&4 allele.

\begin{tabular}{|c|c|c|c|}
\hline (A) & & & \\
\hline \multirow[t]{2}{*}{ A $\beta$-Deposits } & $A \beta$-Negative & A $\beta$-Positive & $p$ \\
\hline & $(\mathrm{N}=10)$ & $(\mathrm{N}=22)$ & \\
\hline Age & $77.5 \pm 6.1$ & $72.3 \pm 7.1$ & 0.054 \\
\hline \multicolumn{4}{|l|}{ Sex } \\
\hline Male & $4(40.0 \%)$ & $9(40.9 \%)$ & $>0.999$ \\
\hline Female & $6(60.0 \%)$ & $13(59.1 \%)$ & \\
\hline Years of education & $9.6 \pm 4.4$ & $13.5 \pm 4.9$ & 0.039 \\
\hline \multicolumn{4}{|l|}{$A P O E$ \&4 allele } \\
\hline Non-carrier & $9(90.0 \%)$ & $4(18.2 \%)$ & 0.001 \\
\hline Carrier & $1(10.0 \%)$ & $18(81.8 \%)$ & \\
\hline $\begin{array}{l}\text { Average SUVR } \\
\text { flutems of }\left[{ }^{18} \mathrm{~F}\right] \\
\text { flumol }\end{array}$ & $0.5 \pm 0.1$ & $0.7 \pm 0.1$ & $<0.001$ \\
\hline
\end{tabular}

\begin{tabular}{lccc}
\hline (B) & & & \\
\hline APOE $\varepsilon$ 4 Allele & Non-Carrier & Carrier & $p$ \\
\hline & $(\mathrm{N}=13)$ & $(\mathrm{N}=19)$ & \\
\hline Age (years) & $75.8 \pm 6.4$ & $72.6 \pm 7.6$ & 0.229 \\
Sex & & & \\
$\quad$ Male & $6(46.2 \%)$ & $7(36.8 \%)$ & 0.873 \\
$\quad$ Female & $7(53.8 \%)$ & $12(63.2 \%)$ & 0.268 \\
Years of education & $11.1 \pm 4.2$ & $13.1 \pm 5.5$ & \\
A $\beta$ deposits & $9(69.2 \%)$ & $1(5.3 \%)$ & 0.001 \\
$\quad$ A $\beta$ neg & $4(30.8 \%)$ & $18(94.7 \%)$ & \\
$\quad$ Average SUVR & $0.6 \pm 0.2$ & $0.7 \pm 0.1$ & 0.003 \\
flutemetamol & &
\end{tabular}

Data are presented as the mean \pm SD unless indicated otherwise. $\mathrm{A} \beta$ Neg, negative deposits of $\mathrm{A} \beta ; \mathrm{A} \beta$ pos, positive deposits of $A \beta$; SUVR $\mathrm{PONS}$, standardized uptake value ratios of $\left[{ }^{18} \mathrm{~F}\right]$ flutemetamol, using pons as a reference region.

There were no significant differences in age, sex, and years of education between patients with MCI with and without the APOE $\varepsilon 4$-allele (Table 1B). We found a higher ratio 
of $\mathrm{A} \beta$ deposits in $A P O E \varepsilon 4$ carriers. APOE $\varepsilon 4$ carriers showed higher average $\mathrm{SUVR}_{\mathrm{PONS}}$ than non-carriers (Table 1B).

\subsection{Changes in Neuropsychological Performance}

For the CERAD-K subdomain and total scores, after adjustment for age, sex, and years of education, the main effect for the $\mathrm{tDCS}, \mathrm{A} \beta$ deposits, and $A P O E$ genotype was not significant. Additionally, there was a nonsignificant interaction between $\mathrm{tDCS}$ and AD risk factors, including APOE genotype and $\mathrm{A} \beta$ deposition.

\subsection{Changes in Functional Segregation and Integration of the DMN: An ROI-Based Analysis}

In terms of functional segregation of the DMN, the main effects of $t D C S, A \beta$ deposits, and $A P O E$ genotype were not significant. Additionally, there was nonsignificant interaction between $\mathrm{tDCS}$ and $\mathrm{A} \beta$ deposition. However, there was an interaction between $\mathrm{tDCS}$ and the $A P O E \varepsilon 4$-allele, which could be attributed to increased temporal pole fALFF after tDCS application in MCI APOE $\varepsilon 4$-allele carriers ( $p=0.036$, Figure 1A). Additionally, this interaction yielded a large effect size (partial $\eta^{2}=0.164$ ). However, we found a nonsignificant association between the baseline average SUVRPONS and change in temporal pole fALFF $(p=0.090)$. Despite not reaching statistical significance, this association showed a large effect size $\left(R^{2}=0.289\right)$.
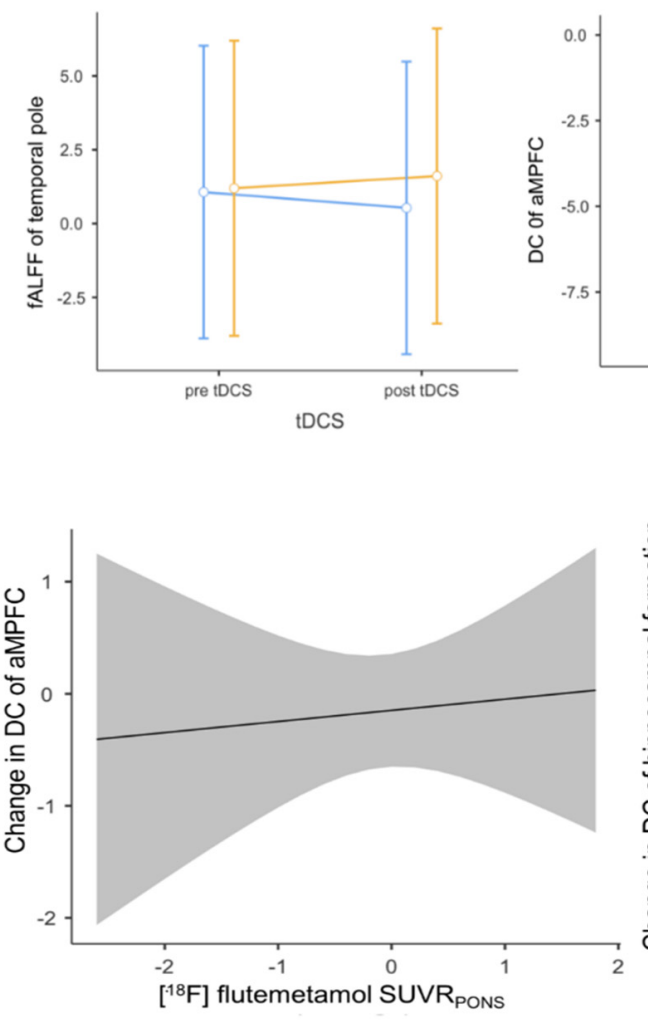

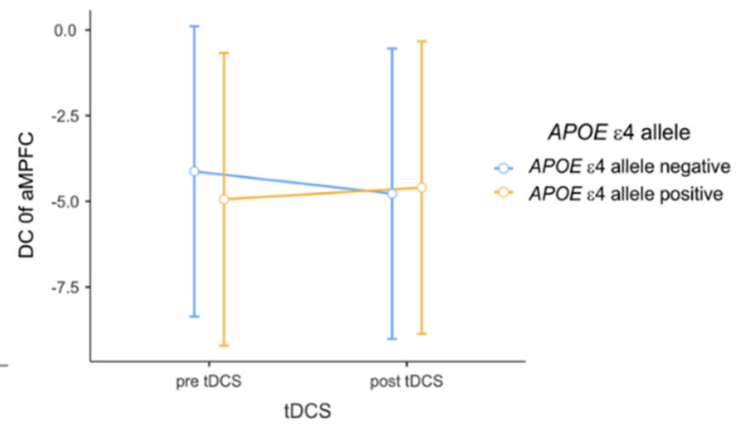

(A)

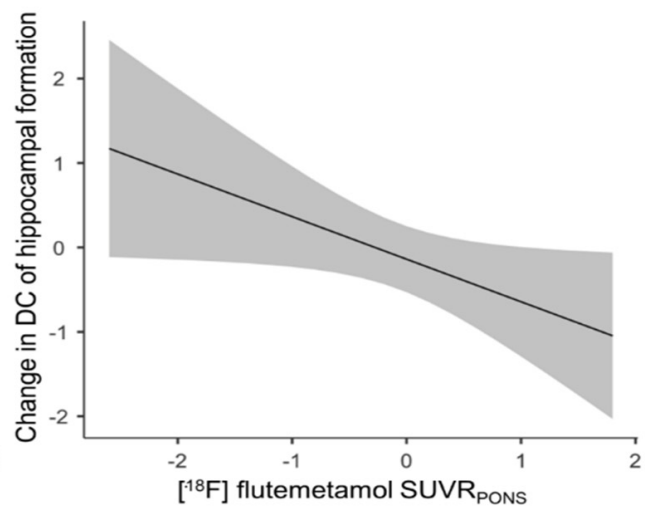

(B)

Figure 1. (A) Impact of interaction between $\mathrm{tDCS}$ and APOE $\varepsilon 4$ allele on $\mathrm{AALFF}$ and DC. (B) Associations between $\left[{ }^{18} \mathrm{~F}\right]$ flutemetamol SUVRPONS and changes in amplitude of DC. Repeated-measures analysis of variance was used to predict the impact of interaction (A) between $\mathrm{tDCS}$ and APOE $\varepsilon 4$ allele on $\mathrm{AALFF}$ and DC, with adjustment for age, sex, and education years ( $p=0.036$, partial $\eta^{2}=0.164 ; p=0.056$, partial $\eta^{2}=0.138$, respectively). (B) Multiple linear regression analysis was used to evaluate the associations between $\left[{ }^{18} \mathrm{~F}\right]$ flutemetamol SUVR $\mathrm{PONS}_{\text {and }}$ changes in DC before and after $\mathrm{tDCS}$, with adjustment for age, sex, and education years $\left(p=0.075, \mathrm{R}^{2}=0.240 ; p=0.042, \mathrm{R}^{2}=0.182\right)$. Each variable was z-transformed using the mean and standard deviation. Changes in FALFF and DC were defined as post-tDCS $z$-transformed values minus pre-tDCS z-transformed values. Abbreviations: tDCS, transcranial direct current stimulation; fALFF, fractional amplitude of low-frequency fluctuation; DC, degree centrality; SUVR, standardized uptake value ratio; A $\beta$, amyloid beta; aMPFC, anterior medial prefrontal cortex. 
Regarding functional integration of the DMN, the main effect of tDCS and AD risk factors was not significant. Although as well as a nonsignificant interaction between tDCS and $\mathrm{AD}$ risk factors, there was a statistical trend toward an interaction between $\mathrm{tDCS}$ and the APOE \&4-allele, possibly attributable to increased aMPFC DC after tDCS application in MCI patients with $\mathrm{A} \beta$ deposits, yielding a large effect size $\left(p=0.056\right.$, partial $\eta^{2}=0.138$, Figure 1A). Additionally, we found a statistical trend toward a positive association between baseline average SUVRPONS and change in aMPFC DC $\left(p=0.075, R^{2}=0.240\right.$, Figure $\left.1 B\right)$, but a negative association between average $S U V R_{P O N S}$ and change in hippocampal formation DC $\left(p=0.042, R^{2}=0.182\right.$, Figure $\left.1 B\right)$, with a large effect size.

\subsection{Changes in Functional Segregation and Integration Parameters: Whole Brain Voxel-Based Analysis}

No brain regions showed a significant impact of tDCS-by-group interaction on the fALFF and DC in each sub-group. Brain regions that showed changes in fALFF after tDCS according to $A P O E$ genotype and $\mathrm{A} \beta$ deposition are displayed in Figure 2A,B. The brain regions that showed significant changes in fALFF differed between MCI APOE $\varepsilon 4$ carriers and non-carriers. Additionally, increased and decreased fALFF values were observed in the right inferior temporal gyrus and crus I of the cerebellum, respectively, after tDCS, in both $\mathrm{MCI}$ patients with and without $\mathrm{A} \beta$ deposition. However, other brain regions that showed significant changes in fALFF also differed between MCI patients with and without $\mathrm{A} \beta$ deposits.

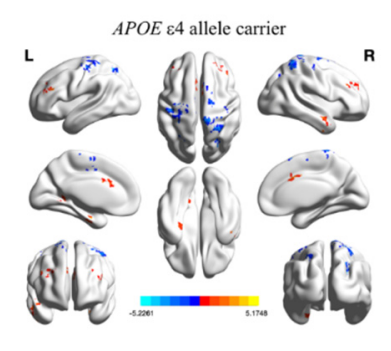

(A)

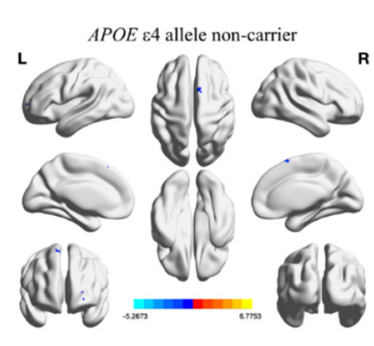

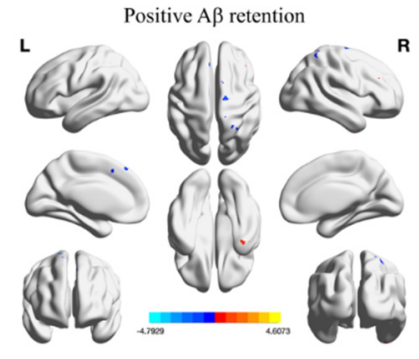

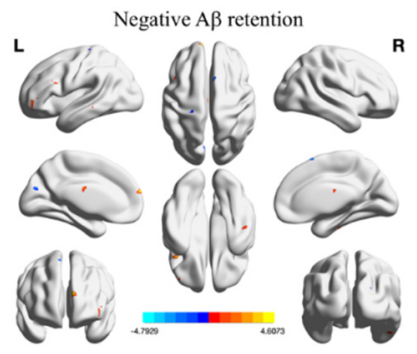

(B)

Figure 2. Significant changes in fALFF after tDCS for patients with mild cognitive impairment (A) with and without the $A P O E \& 4$ allele, and (B) with and without $\mathrm{A} \beta$ deposits. Whole-brain voxel-wise fALFF analysis results. Thresholds were set using GRF correction at a $p$-value of $<0.05$, voxel $p<0.001$. The statistical threshold of the cluster size is described in Figure 2. Abbreviations: tDCS, transcranial direct current stimulation; DC, degree centrality; A $\beta$, amyloid beta.

In terms of functional integration, brain regions that showed changes in DC after tDCS according to APOE genotype and $A \beta$ deposition are shown in Figure $3 \mathrm{~A}, \mathrm{~B}$. The brain regions that showed significant changes in DC differed between MCI APOE4 carriers and non-carriers and patients with and without $A \beta$ deposits. These anatomical regions, their corresponding MNI coordinates, and the intensity of peak points in each cluster are shown in Tables 2 and 3. 


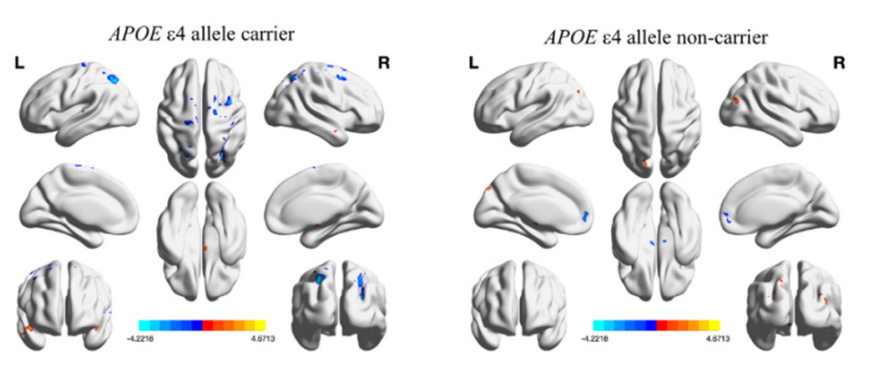

(A)
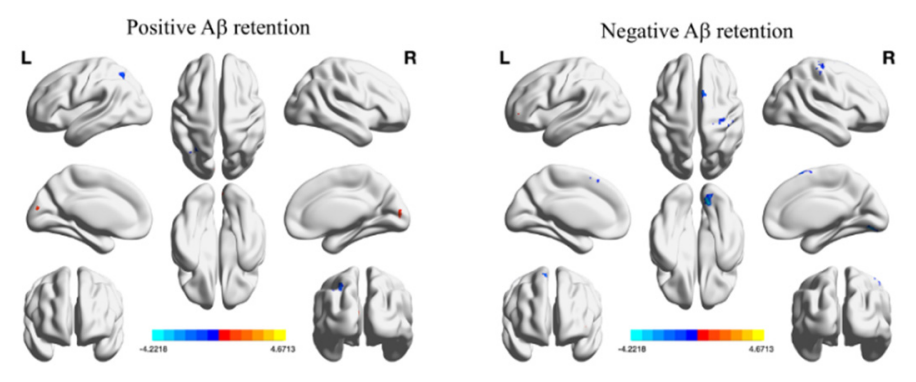

(B)

Figure 3. Significant changes in DC after tDCS of mild cognitive impairment patients (A) with and without the APOE $\varepsilon 4$ allele, and (B) with and without A $\beta$ deposits. Whole-brain voxel-wise fALFF analysis results. Whole-brain voxel-wise DC analysis results. Thresholds were set using GRF correction at a $\mathrm{p}$-value of $<0.05$, voxel $p<0.001$. The statistical threshold of the cluster size is described in Figure 3. Abbreviations: MNI, Montreal Neurological Institute coordinate; aMCI, amnestic mild cognitive impairment group; L/R, left/right; $\mathrm{tDCS}$, transcranial direct current stimulation; fALFF, fractional amplitude of low-frequency fluctuation.

Table 2. Changes in fALFF of MCI patients after tDCS, according to (A) APOE genotype and (B) A $\beta$ deposits.

\begin{tabular}{|c|c|c|c|c|c|c|}
\hline \multicolumn{7}{|l|}{ (A) } \\
\hline Region & $\mathbf{L} / \mathbf{R}$ & Cluster & $\begin{array}{l}\text { Peak } \\
\text { T Value }\end{array}$ & \multicolumn{3}{|c|}{$\begin{array}{l}\text { Peak MNI Coordinates } \\
\qquad(x, y, z)\end{array}$} \\
\hline \multicolumn{7}{|c|}{ Changes in fALFF of MCI $A P O E \varepsilon 4$ Carriers } \\
\hline \multicolumn{7}{|l|}{ tDCS > baseline } \\
\hline Middle temporal gyrus & $\mathrm{R}$ & 98 & 3.9585 & 51 & -3 & -24 \\
\hline Lobule III of cerebellum & $\mathrm{L}$ & 187 & 5.0774 & -3 & -45 & -21 \\
\hline Parahippocampal gyrus & $\mathrm{R}$ & 40 & 3.2239 & 33 & -18 & -30 \\
\hline Precuneus & $\mathrm{L}$ & 41 & 4.831 & -18 & -48 & 3 \\
\hline Inferior frontal gyrus, triangular part & $\mathrm{L}$ & 81 & 3.4026 & -45 & 30 & 24 \\
\hline Middle cingulate gyri & $\mathrm{R}$ & 62 & 3.8016 & 6 & 12 & 36 \\
\hline Midcingulate area & $\mathrm{L}$ & 189 & 3.1684 & -12 & -42 & 51 \\
\hline \multicolumn{7}{|l|}{ tDCS $<$ baseline } \\
\hline Middle occipital gyrus & $\mathrm{R}$ & 45 & -5.1376 & 27 & -75 & 30 \\
\hline Superior frontal gyrus & $\mathrm{L}$ & 384 & -5.2261 & -30 & -3 & 69 \\
\hline Superior parietal gyrus & $\mathrm{R}$ & 250 & -4.3472 & 27 & -51 & 72 \\
\hline Postcentral gyrus & $\mathrm{L}$ & 38 & -4.5553 & -42 & -21 & 57 \\
\hline Supplementary motor area & $\mathrm{R}$ & 43 & -3.7324 & 3 & 18 & 63 \\
\hline Superior frontal gyrus & $\mathrm{R}$ & 61 & -4.9716 & 15 & -9 & 69 \\
\hline \multicolumn{7}{|c|}{ Changes in fALFF of MCI APOE $\varepsilon 4$ Non-Carriers } \\
\hline \multicolumn{7}{|l|}{ tDCS > baseline } \\
\hline Inferior occipital gyrus & $\mathrm{L}$ & 29 & 5.1353 & -42 & -72 & -6 \\
\hline $\begin{array}{l}\text { Calcarine fissure and surrounding cortex } \\
\text { tDCS }<\text { baseline }\end{array}$ & $\mathrm{R}$ & 29 & 3.8547 & 18 & -81 & 9 \\
\hline Superior frontal gyrus, orbital & $\mathrm{L}$ & 47 & -4.0932 & -27 & 57 & -3 \\
\hline Supplementary motor area & $\mathrm{L}$ & 79 & -4.4351 & -3 & 15 & 63 \\
\hline \multicolumn{7}{|l|}{ (B) } \\
\hline Region & $\mathbf{L} / \mathbf{R}$ & Cluster & $\begin{array}{c}\text { Peak } \\
\text { T Value }\end{array}$ & \multicolumn{3}{|c|}{$\begin{array}{l}\text { Peak MNI Coordinates } \\
\qquad(x, y, z)\end{array}$} \\
\hline \multicolumn{7}{|c|}{ Changes in fALFF of MCI Patients with $A \beta$ Deposits } \\
\hline \multicolumn{7}{|l|}{ tDCS $>$ baseline } \\
\hline Inferior temporal gyrus & $\mathrm{R}$ & 61 & 4.0777 & 39 & -6 & -45 \\
\hline Crus I of of cerebellum & $\mathrm{L}$ & 36 & 4.6073 & -15 & -72 & -33 \\
\hline $\begin{array}{l}\text { Lobule III of cerebellum } \\
\text { tDCS }<\text { baseline }\end{array}$ & $\mathrm{L}$ & 62 & 4.374 & -3 & -45 & -21 \\
\hline Crus I of of cerebellum & $\mathrm{R}$ & 40 & -4.5424 & 36 & -81 & -36 \\
\hline
\end{tabular}


Table 2. Cont.

\begin{tabular}{|c|c|c|c|c|c|c|}
\hline Supramarginal gyrus & $\mathrm{R}$ & 38 & -3.4884 & 60 & -30 & 33 \\
\hline Superior parietal gyrus & $\mathrm{R}$ & 99 & -4.3392 & 27 & -51 & 72 \\
\hline Superior frontal gyrus, medial & $\mathrm{R}$ & 146 & -3.5363 & 9 & 33 & 57 \\
\hline Superior frontal gyrus & $\mathrm{L}$ & 119 & -4.3879 & -27 & -9 & 72 \\
\hline Paracentral lobule & $\mathrm{L}$ & 35 & -2.7845 & -6 & -30 & 78 \\
\hline \multicolumn{7}{|c|}{ Changes in fALFF of MCI Patients without $A \beta$ Deposits } \\
\hline \multicolumn{7}{|l|}{ tDCS > baseline } \\
\hline Inferior temporal gyrus & $\mathrm{R}$ & 29 & 4.9944 & 48 & -21 & -27 \\
\hline Middle temporal gyrus & $\mathrm{L}$ & 28 & 6.7989 & -57 & -36 & -6 \\
\hline Middle frontal gyrus & $\mathrm{L}$ & 42 & 6.345 & -39 & 45 & -9 \\
\hline Inferior frontal gyrus triangular part & $\mathrm{L}$ & 32 & 5.266 & -42 & 18 & 6 \\
\hline Superior frontal gyrus, medial & $\mathrm{L}$ & 27 & 7.7112 & -9 & 63 & 21 \\
\hline Precuneus & $\mathrm{R}$ & 32 & 4.4101 & 9 & -60 & 54 \\
\hline \multicolumn{7}{|l|}{ tDCS $<$ baseline } \\
\hline Crus I of cerebellum & $\mathrm{R}$ & 27 & -4.3276 & 48 & -54 & -27 \\
\hline Cuneus & $\mathrm{L}$ & 36 & -4.2645 & -6 & -81 & 24 \\
\hline Superior occipital gyrus & $\mathrm{R}$ & 30 & -4.1793 & 24 & -90 & 27 \\
\hline Middle frontal gyrus & $\mathrm{R}$ & 29 & -5.2138 & 45 & 12 & 42 \\
\hline Supplementary motor area & $\mathrm{R}$ & 27 & -6.8382 & 6 & 15 & 63 \\
\hline Postcentral gyrus & $\mathrm{L}$ & 28 & -3.587 & -24 & -27 & 72 \\
\hline
\end{tabular}

Thresholds were set using GRF correction at a p-value of $<0.05$, voxel $p<0.001$. (A) APOE $\varepsilon 4$ allele carrier, cluster size $>38$; APOE $\varepsilon 4$ allele non-carrier, cluster size $>29$; (B) A $\beta$ deposit-positive, cluster size $>35$; A $\beta$ deposit-negative, cluster size $>27$. Brain regions that showed significant changes are described in Table 2. Abbreviations: tDCS, transcranial direct current stimulation; fALFF, fractional amplitude of low-frequency fluctuation; $\mathrm{A} \beta$, amyloid beta.

Table 3. Changes in DC of MCI patients after tDCS, according to (A) APOE genotype and (B) A $\beta$ deposits.

\begin{tabular}{|c|c|c|c|c|c|c|}
\hline \multicolumn{7}{|l|}{ (A) } \\
\hline Region & $\mathbf{L} / \mathbf{R}$ & Cluster & $\begin{array}{l}\text { Peak } \\
\text { T Value }\end{array}$ & \multicolumn{3}{|c|}{$\begin{array}{l}\text { Peak MNI Coordinates } \\
\qquad(x, y, z)\end{array}$} \\
\hline \multicolumn{7}{|c|}{ Changes in DC of MCI Patients with APOE \&4 Carrier } \\
\hline \multicolumn{7}{|l|}{ tDCS > baseline } \\
\hline Lobule VIIB of cerebellar hemisphere & $\mathrm{R}$ & 78 & 3.7442 & 30 & -72 & -48 \\
\hline Temporal pole: superior temporal gyrus & $\mathrm{R}$ & 115 & 4.3302 & 45 & 3 & -15 \\
\hline Temporal pole: superior temporal gyrus & $\mathrm{L}$ & 108 & 4.3954 & -42 & 3 & -15 \\
\hline $\begin{array}{l}\text { Calcarine fissure and surrounding cortex } \\
\text { tDCS }<\text { baseline }\end{array}$ & $\mathrm{R}$ & 67 & 3.7306 & 9 & -87 & 9 \\
\hline Superior parietal gyrus & $\mathrm{R}$ & 178 & -4.1919 & 27 & -63 & 51 \\
\hline Superior parietal gyrus & $\mathrm{L}$ & 767 & -5.1001 & -24 & -69 & 48 \\
\hline \multicolumn{7}{|c|}{ Changes in DC of MCI Patients with APOE $\varepsilon 4$ Non-Carrier } \\
\hline \multicolumn{7}{|l|}{ tDCS > baseline } \\
\hline Inferior temporal gyrus & $\mathrm{L}$ & 48 & 4.5971 & -42 & -60 & -6 \\
\hline Middle occipital gyrus & $\mathrm{R}$ & 60 & 4.2846 & 33 & -78 & 30 \\
\hline $\begin{array}{l}\text { Middle occipital gyrus } \\
\text { tDCS < baseline }\end{array}$ & $\mathrm{L}$ & 84 & 4.7948 & -15 & -81 & 39 \\
\hline Superior frontal gyrus, medial & $\mathrm{L}$ & 338 & -6.6552 & 0 & 57 & 0 \\
\hline \multicolumn{7}{|l|}{ (B) } \\
\hline Region & $\mathbf{L} / \mathbf{R}$ & Cluster & $\begin{array}{c}\text { Peak } \\
\text { T Value }\end{array}$ & \multicolumn{3}{|c|}{$\begin{array}{l}\text { Peak MNI Coordinates } \\
\qquad(x, y, z)\end{array}$} \\
\hline \multicolumn{7}{|c|}{ Changes in DC of MCI Patients with A $\beta$ Deposits } \\
\hline \multicolumn{7}{|l|}{ tDCS $>$ baseline } \\
\hline Lobule VIII of cerebellum & $\mathrm{R}$ & 70 & 3.9953 & 12 & -66 & -45 \\
\hline $\begin{array}{l}\text { Calcarine fissure and surrounding cortex } \\
\text { tDCS }<\text { baseline }\end{array}$ & $\mathrm{R}$ & 230 & 4.6267 & 9 & -87 & 9 \\
\hline Superior parietal gyrus & $\mathrm{L}$ & 76 & -3.427 & -21 & -51 & 45 \\
\hline
\end{tabular}


Table 3. Cont.

\begin{tabular}{|c|c|c|c|c|c|c|}
\hline \multicolumn{7}{|c|}{ Changes in DC of MCI Patients without $A \beta$ Deposits } \\
\hline \multicolumn{7}{|l|}{ tDCS $>$ baseline } \\
\hline Superior frontal gyrus, medial orbital & $\mathrm{R}$ & 53 & 4.7797 & 12 & 66 & -9 \\
\hline Middle frontal gyrus & $\mathrm{L}$ & 70 & 4.8997 & -48 & 42 & 15 \\
\hline $\begin{array}{l}\text { Middle temporal gyrus } \\
\text { tDCS < baseline }\end{array}$ & $\mathrm{R}$ & 53 & 4.9548 & 66 & -48 & 9 \\
\hline Lingual gyrus & $\mathrm{R}$ & 55 & -5.8024 & 12 & -81 & -12 \\
\hline Superior frontal gyrus, medial & $\mathrm{L}$ & 48 & -4.093 & 0 & 57 & 0 \\
\hline Lenticular nucleus, Putamen & $\mathrm{L}$ & 48 & -4.2643 & -18 & 3 & 6 \\
\hline Middle occipital gyrus & $\mathrm{L}$ & 50 & -3.9537 & -24 & -84 & 9 \\
\hline Supplementary motor area & $\mathrm{R}$ & 115 & -3.9209 & 12 & 12 & 69 \\
\hline Precentral gyrus & $\mathrm{L}$ & 59 & -3.5792 & -33 & -21 & 72 \\
\hline Postcentral gyrus & $\mathrm{R}$ & 153 & -5.5196 & 27 & -30 & 60 \\
\hline
\end{tabular}

Whole-brain voxel-wise DC analysis results. Thresholds were set using GRF correction at a $p$-value of $<0.05$, voxel $p<0.001$. (A) $A P O E \varepsilon 4$ allele carrier, cluster size $>62 ; A P O E \varepsilon 4$ allele non-carrier, cluster size $>48$; (B) A $\beta$ deposit-positive, cluster size $>52$; A $\beta$ deposit-negative, cluster size $>48$. Brain regions that showed significant changes are described in Table 3. Abbreviations: MNI, Montreal Neurological Institute coordinate; aMCI, amnestic mild cognitive impairment group; L/R, left/right; tDCS, transcranial direct current stimulation; fALFF, fractional amplitude of low-frequency fluctuation.

\section{Discussion}

The current study aimed to evaluate the impact of anodal-tDCS on cognitive performance and functional segregation and integration parameters in MCI patients, according to the presence of $\mathrm{A} \beta$ deposits and the APOE $\varepsilon 4$-allele. We evaluated the effect of interactions between anodal-tDCS application and AD risk factors on changes in cognitive function and intrinsic brain activity and explored differences in changes in cognitive function and spontaneous brain activity parameters between MCI patients with and without AD risk factors after multiple sequential anodal-tDCS sessions.

\subsection{Changes in Neuropsychological Performance}

With regard to cognitive performance, the impact of tDCS was not significant for changes in cognitive performance. We also found a nonsignificant interaction between anodal-tDCS and AD risk factors in the current study.

Similarly, in a previous study that conducted a nine-session clinical trial for 3 weeks in MCI patients, there was no improvement in the objective neuropsychological test score [17]. On the other hand, some prior studies have demonstrated improvement of semantic wordretrieval performance after a single-session anodal-tDCS application over the left ventral inferior frontal gyrus of MCI patients [16]. In previous studies that performed anodal-tDCS on AD patients, they reported improved MMSE scores [10], recognition memory [11], and global functioning as compared to the sham group [12]. Additionally, in a meta-analysis of administering $\mathrm{tDCS}$ in patients with mild to moderate AD, repeated-session tDCS was not significantly more effective than single-session tDCS [38]. Moreover, stimulation of the temporal cortex significantly improved cognitive function, as compared to other areas, although the left DLPFC was the most frequently stimulated area [38]. The tDCS protocol of the present study did not contain factors that show beneficial effects identified in the meta-analysis, which could contribute to the restricted improvement in cognitive function. However, this meta-analysis targeted only seven studies, and the sample size was small, and thus results should be interpreted cautiously. Additionally, the insufficient sample size of the current pilot study could contribute to the nonsignificant change in cognitive function.

\subsection{Changes in Functional Segregation and Integration of the DMN: An ROI-Based Analysis}

With regard to changes in brain functional segregation parameters, this study found a significant interaction between $\mathrm{tDCS}$ and $A P O E$ \&4-allele in the left temporal pole. This interaction could contribute to increased temporal pole fALFF after anodal-tDCS applica- 
tion in MCI patients. The left temporal pole is part of the DMPFC subsystem of the DMN, which is vulnerable to AD pathology [35]. The DC of the left temporal lobe is lower in patients with MCI than in cognitively intact older adults [39]. Additionally, the temporal pole was associated with an abnormal insula network in MCI patients, and decreased functional connectivity in this network is related to cognitive decline in MCI patients [40]. Furthermore, the $A P O E \varepsilon 4$-allele reduces connectivity of the hippocampal network, which includes the temporal pole in healthy older adults [26]. Although the present study showed a relative lack of evidence for functional integration changes, application of anodal-tDCS in prodromal AD patients with high-risk factors appears to restore the local intrinsic change in the temporal pole found in the MCI stage. This observation might support the hypothesis that tDCS-induced improvement is related to the restoration, rather than compensation, of brain activity patterns [41].

In this study, although there was a lack of a statistical significance, the index reflecting the global functional integration of aMPFC also showed a similar pattern to the interaction found in the functional segregation parameter of the temporal pole, yielding a large effect size. These results might be attributed to increased functional integration after anodal-tDCS application in MCI patients with the APOE $\varepsilon 4$ genotype. The aMPFC is an anterior core set of hubs in the DMN and shows global connectivity with other areas that constitute a DMN subsystem [35]. Additionally, the anterior DMN shows increased connectivity during $\mathrm{AD}$ and cognitive decline progression, and this change in the anterior hubs may be a compensatory response to AD pathology [42]. However, it is possible that these results may underestimate $\mathrm{A} \beta$-mediated hyperactivation in the early stages of $\mathrm{AD}$ [43]. Therefore, it is important to bear in mind the possible bias in these responses.

Another important finding was that a decreased change in DC of hippocampal formation was exhibited in the higher baseline $A \beta$ deposits with a large effect size. This result might reflect decoupling of the hippocampal formation from posterior DMN nodes at the prodromal AD stage [44], and it is estimated that the tDCS application does not significantly affect pathologic functional changes in the hippocampal formation.

\subsection{Changes in Functional Segregation and Integration Parameters: Whole Brain Voxel-Based Analysis}

Lastly, in the present study, differences were observed in changing functional segregation and integration patterns after anodal-tDCS application, depending on the APOE $\varepsilon 4$-allele or $\mathrm{A} \beta$ deposits by whole-brain voxel-based analysis in MCI patients. In terms of functional segregation parameters after anodal-tDCS application, our MCI patients with $A P O E \varepsilon 4$-allele displayed increased local intrinsic brain activity in DMN hub regions and AD compensatory regions, in which previous studies have shown a decreasing trend of fALFF across the AD spectrum [45]. However, MCI patients without the APOE $\varepsilon 4$-allele showed increased fALFF after repetitive anodal-tDCS administration in different brain regions, such as the inferior occipital gyrus, calcarine fissure, and surrounding cortex. The inferior occipital gyrus has been documented to be vulnerable during the MCI stage and is connected with deep brain structures related to MCI pathology [46]. Additionally, the fALFF of the calcarine fissure and surrounding cortex showed a decreasing trend during the AD course [47]. However, the lack of information on the APOE genotype in previous reports adds further caution regarding the interpretation of these findings. In MCI patients in the present study, regional intrinsic activity of the inferior temporal gyrus was increased both with and without $A \beta$ deposits, and this region has shown lower local integrity in the MCI group than in the normal group in our previous study [48]. Furthermore, the cerebellum, in which regional intrinsic brain activity increased after tDCS in MCI patients with $\mathrm{A} \beta$ deposits, was also the area in which fALFF tended to decrease with AD progression in a previous study [45]. Therefore, these findings might indicate that increased fALFF in functionally deteriorated regions might be induced by sequential anodal-tDCS during the prodromal AD stage. Additionally, MCI patients without $\mathrm{A} \beta$ deposits showed increased intrinsic brain activity at various locations in the frontal gyrus, unlike those with $\mathrm{A} \beta$ deposition after multiple sessions of anodal-tDCS. In a prior study, the frontal cortex 
showed hypermetabolism in MCI patients without $\mathrm{A} \beta$ accumulation, and MCI patients with cortical hypermetabolism did not convert to AD during the follow-up period [49]. Hence, it could conceivably be hypothesized that sequential anodal-tDCS may restore spontaneous brain activity in MCI patients with $A \beta$ deposits but play a compensatory role in those without $A \beta$ deposition. Future studies on the current topic are therefore recommended.

Regarding the functional integration parameter evaluated by whole-brain voxel-based analysis, we found that $\mathrm{MCI}$ patients with $\mathrm{AD}$ risk factors showed increased DC in the cerebellum after anodal-tDCS, similar to the pattern of functional segregation parameter changes. Another finding was that $\mathrm{MCI}$ patients with the APOE $\varepsilon 4$-allele showed increased temporal pole DC after anodal-tDCS, in which a fALFF increase was observed in ROI-based analysis. According to these data, it might be assumed that the intensity at which a region locally activated by anodal-tDCS is integrated with other regions increases simultaneously in MCI patients with high-risk factors of AD.

\subsection{Limitations}

A significant limitation of the current pilot study is that the sample size was relatively small, and no comparisons with a sham group were made. Consequently, there is a relative lack of statistical robustness for the interaction between anodal-tDCS application and $\mathrm{AD}$ risk factors for changes in cognitive function and brain functional segregation and integration. Lastly, considering the after-effects of tDCS [9] and the important role of stimulation frequency for outcomes in $\mathrm{MCI}$ and $\mathrm{AD}$ patients [50], further research, applying tDCS for a longer duration, is needed.

\section{Conclusions}

This study provides an initial step in searching for conditions that may deliver optimal effects when tDCS is administered during the AD prodromal stage. It is necessary to identify the preventive and therapeutic mechanisms of tDCS in AD more clearly, and to establish a foundation for precision medicine for tDCS treatment of AD.

Supplementary Materials: The following are available online at https://www.mdpi.com/article/10 .3390 /brainsci11060772/s1, Table S1: Regions-of-interest (ROIs) in the default network.

Author Contributions: D.-W.K.: conceptualization, methodology, data curation, writing-original draft, visualization, formal analysis, funding acquisition. S.-M.W.: methodology, data curation, writing-review and editing. T.-Y.K.: software, investigation. D.K.: software, investigation. H.-R.N.: investigation, visualization. N.-Y.K.: methodology, data curation. C.-U.L.: conceptualization, supervision. H.-K.L.: conceptualization, methodology, writing-review and editing, supervision, project administration. All authors have read and agreed to the published version of the manuscript.

Funding: This work was supported by a National Research Foundation of Korea (NRF) grant funded by the Korean government (Ministry of Science and ICT) (No. 2019R1C1C1007608). The funders had no role in study design, data collection and analysis, decision to publish, or preparation of the manuscript.

Institutional Review Board Statement: The study was conducted according to the guidelines of the Declaration of Helsinki, and approved by the Institutional Review Board of the Catholic University of Korea, which approved all research activity (protocol code: SC19DEST0012 and date of approval: 16 April 2019).

Informed Consent Statement: Informed consent was obtained from all subjects.

Data Availability Statement: The datasets generated or analyzed during the current study are not publicly available due to Patient Data Management Protocol of Yeouido St. Mary's Hospital but are available from the corresponding author on reasonable request.

Conflicts of Interest: The authors declare no conflict of interest. 


\section{References}

1. Petersen, R.C.; Negash, S. Mild Cognitive Impairment: An Overview. CNS Spectr. 2008, 13, 45-53. [CrossRef]

2. Gauthier, S.; Reisberg, B.; Zaudig, M.; Petersen, R.C.; Ritchie, K.; Broich, K.; Belleville, S.; Brodaty, H.; Bennett, D.; Chertkow, H.; et al. Mild cognitive impairment. Lancet 2006, 367, 1262-1270. [CrossRef]

3. Jean, L.; Bergeron, M.-Ë.; Thivierge, S.; Simard, M. Cognitive Intervention Programs for Individuals With Mild Cognitive Impairment: Systematic Review of the Literature. Am. J. Geriatr. Psychiatry 2010, 18, 281-296. [CrossRef]

4. Lautenschlager, N.T.; Cox, K.; Kurz, A.F. Physical Activity and Mild Cognitive Impairment and Alzheimer's Disease. Curr. Neurol. Neurosci. Rep. 2010, 10, 352-358. [CrossRef]

5. Singh, B.; Parsaik, A.K.; Mielke, M.M.; Erwin, P.J.; Knopman, D.S.; Petersen, R.C.; Roberts, R.O. Association of mediterranean diet with mild cognitive impairment and Alzheimer's disease: A systematic review and meta-analysis. J. Alzheimer's Dis. 2014, 39, 271-282. [CrossRef]

6. Coley, N.; Ngandu, T.; Lehtisalo, J.; Soininen, H.; Vellas, B.; Richard, E.; Kivipelto, M.; Andrieu, S.; Hatice, F.; Van Gool, P.; et al. Adherence to multidomain interventions for dementia prevention: Data from the FINGER and MAPT trials. Alzheimer's Dement. 2019, 15, 729-741. [CrossRef] [PubMed]

7. Liu, C.S.; Rau, A.; Gallagher, D.; Rajji, T.K.; Lanctôt, K.L.; Herrmann, N. Using transcranial direct current stimulation to treat symptoms in mild cognitive impairment and Alzheimer's disease. Neurodegener. Dis. Manag. 2017, 7, 317-329. [CrossRef] [PubMed]

8. Nitsche, M.A.; Fricke, K.; Henschke, U.; Schlitterlau, A.; Liebetanz, D.; Lang, N.; Henning, S.; Tergau, F.; Paulus, W. Pharmacological modulation of cortical excitability shifts induced by transcranial direct current stimula-tion in humans. J. Physiol. 2003, 553, 293-301. [CrossRef] [PubMed]

9. Hansen, N. Action mechanisms of transcranial direct current stimulation in Alzheimer's disease and memory loss. Front. Psychiatry 2012, 3, 48. [CrossRef] [PubMed]

10. Ferrucci, R.; Mameli, F.; Guidi, I.; Mrakic-Sposta, S.; Vergari, M.; Marceglia, S.; Cogiamanian, F.; Barbieri, S.; Scarpini, E.; Priori, A. Transcranial direct current stimulation improves recognition memory in Alzheimer disease. Neurology 2008, 71, 493-498. [CrossRef] [PubMed]

11. Boggio, P.S.; Khoury, L.P.; Martins, D.C.S.; Martins, O.E.M.S.; De Macedo, E.C.; Fregni, F. Temporal cortex direct current stimulation enhances performance on a visual recognition memory task in Alzheimer disease. J. Neurol. Neurosurg. Psychiatry 2008, 80, 444-447. [CrossRef]

12. Khedr, E.M.; Gamal, N.F.; El-Fetoh, N.A.; Khalifa, H.; Ahmed, E.M.; Ali, A.M.; Noaman, M.; El-Baki, A.A.; Karim, A.A. A double-blind randomized clinical trial on the efficacy of cortical direct current stimulation for the treatment of Alzheimer's disease. Front. Aging Neurosci. 2014, 6, 275. [CrossRef] [PubMed]

13. Bystad, M.; Grønli, O.; Rasmussen, I.D.; Gundersen, N.; Nordvang, L.; Wang-Iversen, H.; Aslaksen, P.M. Transcranial direct current stimulation as a memory enhancer in patients with Alzheimer's disease: A randomized, placebo-controlled trial. Alzheimer's Res. Ther. 2016, 8, 1-7. [CrossRef] [PubMed]

14. Gutchess, A.H.; Welsh, R.C.; Hedden, T.; Bangert, A.; Minear, M.; Liu, L.L.; Park, D.C. Aging and the neural correlates of successful picture encoding: Frontal activations compensate for de-creased medial-temporal activity. J. Cogn. Neurosci. 2005, 17, 84-96. [CrossRef]

15. Edin, F.; Klingberg, T.; Johansson, P.; McNab, F.; Tegner, J.; Compte, A. Mechanism for top-down control of working memory capacity. Proc. Natl. Acad. Sci. USA 2009, 106, 6802-6807. [CrossRef] [PubMed]

16. Meinzer, M.; Lindenberg, R.; Phan, M.T.; Ulm, L.; Volk, C.; Flöel, A. Transcranial direct current stimulation in mild cognitive impairment: Behavioral effects and neural mechanisms. Alzheimer's Dement. 2015, 11, 1032-1040. [CrossRef]

17. Yun, K.; Song, I.-U.; Chung, Y.-A. Changes in cerebral glucose metabolism after 3 weeks of noninvasive electrical stimu-lation of mild cognitive impairment patients. Alzheimer's Res. Ther. 2016, 8, 1-9.

18. Sporns, O. Network attributes for segregation and integration in the human brain. Curr. Opin. Neurobiol. 2013, $23,162-171$. [CrossRef]

19. Zou, Q.-H.; Zhu, C.-Z.; Yang, Y.; Zuo, X.-N.; Long, X.-Y.; Cao, Q.-J.; Wang, Y.-F. An improved approach to detection of amplitude of low-frequency fluctuation (ALFF) for resting-state fMRI: Fractional ALFF. J. Neurosci. Methods 2008, 172, 137-141. [CrossRef]

20. Guo, Z.; Liu, X.; Hou, H.; Wei, F.; Liu, J.; Chen, X. Abnormal degree centrality in Alzheimer's disease patients with depression: A resting-state functional magnetic resonance imaging study. Exp. Gerontol. 2016, 79, 61-66. [CrossRef]

21. Buckner, R.L.; Andrews-Hanna, J.R.; Schacter, D.L. The brain's default network: Anatomy, function, and relevance to disease. Ann. N. Y. Acad. Sci. 2008, 1124, 1-38. [CrossRef]

22. Binnewijzend, M.A.; Schoonheim, M.M.; Sanz-Arigita, E.; Wink, A.M.; van der Flier, W.M.; Tolboom, N.; Adriaanse, S.M.; Damoiseaux, J.S.; Scheltens, P.; van Berckel, B.N.; et al. Resting-state fMRI changes in Alzheimer's disease and mild cognitive impairment. Neurobiol. Aging 2012, 33, 2018-2028. [CrossRef]

23. Zeng, M.; Wang, L.; Cheng, B.; Qi, G.; He, J.; Xu, Z.; Han, T.; Liu, C.; Wang, Y. Transcutaneous spinal cord direct current stimulation modulates functional activity and integration in idio-pathic restless legs syndrome. Front. Neurosci. 2020, 14, 873. [CrossRef]

24. Hedden, T.; Van Dijk, K.; Becker, J.A.; Mehta, A.; Sperling, R.A.; Johnson, K.A.; Buckner, R.L. Disruption of Functional Connectivity in Clinically Normal Older Adults Harboring Amyloid Burden. J. Neurosci. 2009, 29, 12686-12694. [CrossRef] 
25. Lim, Y.Y.; Ellis, K.A.; Pietrzak, R.H.; Ames, D.; Darby, D.; Harrington, K.; Martins, R.N.; Masters, C.L.; Rowe, C.; Savage, G.; et al. Stronger effect of amyloid load than APOE genotype on cognitive decline in healthy older adults. Neurology 2012, 79, 1645-1652. [CrossRef] [PubMed]

26. Trachtenberg, A.J.; Filippini, N.; Ebmeier, K.P.; Smith, S.M.; Karpe, F.; Mackay, C.E. The effects of APOE on the functional architecture of the resting brain. NeuroImage 2012, 59, 565-572. [CrossRef]

27. Christensen, H.; Batterham, P.J.; MacKinnon, A.J.; Jorm, A.F.; Mack, H.A.; Mather, K.A.; Anstey, K.J.; Sachdev, P.S.; Easteal, S. The association of APOE genotype and cognitive decline in interaction with risk factors in a 65-69 year old community sample. BMC Geriatr. 2008, 8, 14. [CrossRef]

28. Kemppainen, N.; Johansson, J.; Teuho, J.; Parkkola, R.; Joutsa, J.; Ngandu, T.; Solomon, A.; Stephen, R.; Liu, Y.; Hänninen, T.; et al. Brain amyloid load and its associations with cognition and vascular risk factors in FINGER Study. Neurology 2017, 90, e206-e213. [CrossRef]

29. Berkowitz, C.L.; Mosconi, L.; Rahman, A.; Scheyer, O.; Hristov, H.; Isaacson, R.S. Clinical application of APOE in Alzheimer's prevention: A precision medicine approach. J. Prev. Alzheimer's Dis. 2018, 5, 1-7. [CrossRef]

30. Lee, J.H.; Lee, K.U.; Lee, D.Y.; Kim, K.W.; Jhoo, J.H.; Kim, J.H.; Lee, K.H.; Kim, S.Y.; Han, S.H.; Woo, J.I. Development of the Korean Version of the Consortium to Establish a Registry for Alzheimer's Disease As-sessment Packet (CERAD-K) clinical and neuropsychological assessment batteries. J. Gerontol. Ser. B Psychol. Sci. Soc. Sci. 2002, 57, P47-P53.

31. Park, J.-H. Standardization of Korean version of the Mini-Mental State Examination (MMSE-K) for use in the elderly. Part II. Diagnostic validity. J. Korean Neuropsychiatr. Assoc. 1989, 28, 508-513.

32. Petersen, R.C. Mild cognitive impairment as a diagnostic entity. J. Intern. Med. 2004, 256, 183-194. [CrossRef] [PubMed]

33. Yan, C.; Zang, Y. DPARSF: A MATLAB toolbox for "pipeline" data analysis of resting-state fMRI. Front. Syst. Neurosci. 2010, 4, 13. [CrossRef] [PubMed]

34. Zuo, X.-N.; Ehmke, R.; Mennes, M.; Imperati, D.; Castellanos, F.; Sporns, O.; Milham, M.P. Network Centrality in the Human Functional Connectome. Cereb. Cortex 2012, 22, 1862-1875. [CrossRef]

35. Andrews-Hanna, J.R.; Reidler, J.S.; Sepulcre, J.; Poulin, R.; Buckner, R.L. Functional-anatomic fractionation of the brain's default network. Neuron 2010, 65, 550-562. [CrossRef]

36. Thurfjell, L.; Lilja, J.; Lundqvist, R.; Buckley, C.; Smith, A.; Vandenberghe, R.; Sherwin, P. Automated Quantification of 18FFlutemetamol PET Activity for Categorizing Scans as Negative or Positive for Brain Amyloid: Concordance with Visual Image Reads. J. Nucl. Med. 2014, 55, 1623-1628. [CrossRef]

37. Bansal, R.; Peterson, B.S. Cluster-level statistical inference in fMRI datasets: The unexpected behavior of random fields in high dimensions. Magn. Reson. Imaging 2018, 49, 101-115. [CrossRef]

38. Cai, M.; Guo, Z.; Xing, G.; Peng, H.; Zhou, L.; Chen, H.; McClure, M.A.; He, L.; Xiong, L.; He, B.; et al. Transcranial Direct Current Stimulation Improves Cognitive Function in Mild to Moderate Alzheimer Disease. Alzheimer Dis. Assoc. Disord. 2019, 33, 170-178. [CrossRef]

39. Jacini, F.; Sorrentino, P.; Lardone, A.; Rucco, R.; Baselice, F.; Cavaliere, C.; Aiello, M.; Orsini, M.; Iavarone, A.; Manzo, V.; et al. Amnestic Mild Cognitive Impairment Is Associated With Frequency-Specific Brain Network Alterations in Temporal Poles. Front. Aging Neurosci. 2018, 10, 400. [CrossRef]

40. Xie, C.; Bai, F.; Yu, H.; Shi, Y.; Yuan, Y.; Chen, G.; Li, W.; Chen, G.; Zhang, Z.; Li, S.-J. Abnormal insula functional network is associated with episodic memory decline in amnestic mild cognitive impairment. NeuroImage 2012, 63, 320-327. [CrossRef]

41. Nyberg, L.; Lövdén, M.; Riklund, K.; Lindenberger, U.; Bäckman, L. Memory aging and brain maintenance. Trends Cogn. Sci. 2012, 16, 292-305. [CrossRef]

42. Jones, D.T.; Machulda, M.M.; Vemuri, P.; McDade, E.M.; Zeng, G.; Senjem, M.L.; Gunter, J.L.; Przybelski, S.A.; Avula, R.T.; Knopman, D.S.; et al. Age-related changes in the default mode network are more advanced in Alzheimer disease. Neurology 2011, 77, 1524-1531. [CrossRef]

43. Zott, B.; Simon, M.M.; Hong, W.; Unger, F.; Chen-Engerer, H.J.; Frosch, M.P.; Sakmann, B.; Walsh, D.M.; Konnerth, A. A vicious cycle of $\beta$ amyloid-dependent neuronal hyperactivation. Science 2019, 365, 559-565. [CrossRef]

44. Dillen, K.N.H.; Jacobs, H.I.L.; Kukolja, J.; Richter, N.; Von Reutern, B.; Onur, O.; Langen, K.-J.; Fink, G.R. Functional Disintegration of the Default Mode Network in Prodromal Alzheimer's Disease. J. Alzheimer's Dis. 2017, 59, 169-187. [CrossRef]

45. Yang, L.; Yan, Y.; Wang, Y.; Hu, X.; Lu, J.; Chan, P.; Yan, T.; Han, Y. Gradual Disturbances of the Amplitude of Low-Frequency Fluctuations (ALFF) and Fractional ALFF in Alzheimer Spectrum. Front. Neurosci. 2018, 12, 975. [CrossRef] [PubMed]

46. Liu, J.; Zhang, B.; Wilson, G.; Kong, J.; Initiative, T.A.D.N.; Weiner, M.W.; Aisen, P.; Petersen, R.; Jack, C.R.J.; Jagust, W.; et al. New Perspective for Non-invasive Brain Stimulation Site Selection in Mild Cognitive Impairment: Based on Meta- and Functional Connectivity Analyses. Front. Aging Neurosci. 2019, 11, 228. [CrossRef] [PubMed]

47. Yang, L.; Yan, Y.; Li, Y.; Hu, X.; Lu, J.; Chan, P.; Yan, T.; Han, Y. Frequency-dependent changes in fractional amplitude of lowfrequency oscillations in Alzheimer's disease: A resting-state fMRI study. Brain Imaging Behav. 2020, 14, 2187-2201. [CrossRef] [PubMed]

48. Kang, D.W.; Lim, H.K.; Joo, S.-H.; Lee, N.R.; Lee, C.-U. Alterations in Intra- and Interregional Intrinsic Brain Connectivity Are Differentially Associated with Memory Performance in Amnestic Mild Cognitive Impairment. Dement. Geriatr. Cogn. Disord. 2018, 46, 229-242. [CrossRef] 
49. Ashraf, A.; Fan, Z.; Brooks, D.J.; Edison, P. Cortical hypermetabolism in MCI subjects: A compensatory mechanism? Eur. J. Nucl. Med. Mol. Imaging 2015, 42, 447-458. [CrossRef]

50. Batsikadze, G.; Moliadze, V.; Paulus, W.; Kuo, M.-F.; Nitsche, M.A. Partially non-linear stimulation intensity-dependent effects of direct current stimulation on motor cortex excitability in humans. J. Physiol. 2013, 591, 1987-2000. [CrossRef] 